\title{
Footprints of Airborne Ultrafine Particles on Urban Air Quality and Public Health
}

\section{Prashant Kumar}

Division of Civil, Chemical and Environmental Engineering, Faculty of Engineering and Physical Sciences (FEPS), University of Surrey, GU2 7XH Guildford, United Kingdom

Environmental Flow (EnFlo) Research Centre, FEPS, University of Surrey, GU2 7XH Guildford, United Kingdom

Keywords: Ultrafine particles; Indoor air quality; Outdoor air pollution; Public exposure; Number and size distributions

Emergence of airborne ultrafine particles (UFPs; those below $100 \mathrm{~nm}$ in diameter) in both indoor and outdoor urban settings is continuously attracting attention of the air quality science and management communities worldwide due to their probable adverse impacts on human health and the environment. A simple mean to visualise their tiny size is that the width of a single human hair can accommodate, in parallel, 60,000 and 600 particles of 1 and $100 \mathrm{~nm}$ diameter, respectively. An exponential increase in number of UFP studies has been noticed during the last decade, suggesting a notable advancement in the area of their measurements, physico-chemical characterisation, dispersion modelling and exposure assessment [1]. However, this progress is not yet adequate enough to inform regulatory decision making on a particle number basis. The first question in this context can be raised that where do these UFPs come from and why might we need to control them? Further question can be asked: if the UFPs are needed to be controlled, what are the practical and technical challenges in envisaging control measures? This article revolves around these questions and makes an attempt to highlight recent advances in our understanding and future research priorities. The subsequent paragraphs focuses on the concerns related to outdoor UFPs in urban settings. For the completeness of the article, the last few sections very briefly introduce the UFP related issues in the context of indoor environments.

One of the prominent reasons to pay the UFPs a serious attention is their links with the negative health impacts. The exact biological mechanism through which the UFPs affect the human health is still indecisive but the toxicological and epidemiological evidences have heaped over the past years to demonstrate their adverse impacts on the public health [1]. The minuscule size of UFPs results in significantly large surface area and particle number concentrations (PNCs) but very little particle mass concentrations, leaving them out of current mass based regulatory limits for ambient coarse particulate matter (i.e. $\mathrm{PM}_{2.5}$ and $\mathrm{PM}_{10}$ ). Moreover, freshly emitted UFPs contain toxic contents (e.g. metals, organics) and their tiny size offers them the ability to penetrate the epithelial cells and subsequently accumulate in lymph nodes. Recent studies have indicated that the UFPs carry a potential for oxidative damage to DNA which may lead to increased risk of cancer pathways (see e.g. Kumar et al. [1,2] and references therein). UFPs also play an important role to the fading of urban visibility and global climate change via the coagulation and condensation on to the surfaces of coarse particles [2] and hence contributing to their further growth and modifying optical and radiative properties.

In the outdoor environments, the UFPs originate from both natural (e.g. atmospheric formation, biogenic or geogenic) and anthropogenic (e.g. combustion) activities. The latter activities, as discussed here, are of particular concern due to a peculiar coexistence of both the attendance of dominant source (i.e. burning of fossil fuels in internal combustion engines) in urban areas and exposure to a greater number of inhabitants. For instance, road vehicles can alone contribute up to $90 \%$ of total PNCs in polluted urban environments. As far as the fraction of PNCs in different size is concerned, about $99 \%$ of total PNCs generally fall below $300 \mathrm{~nm}$ diameter and over $~ 80 \%$ in UFP size range. Generally, majority of the PNCs are contributed by particles below $100 \mathrm{~nm}$ but opposite is the case for particle mass concentrations as bulk of the mass is added by particles over $100 \mathrm{~nm}$. This suggests that both these metrics (number and mass), especially for UFPs, are not complimentary and targeted control measures are required [3].

Non-exhaust emission sources are the other less explored anthropogenic sources. Some of these sources include brake and tyre wear, stack emissions from power plants and waste incineration, idling, taxiing and take-off from aircraft at airports, ship journeys from ports or harbours, construction and demolition activities, cooking in restaurants, biomass burning, fuel combustion during gardening, agriculture processes, cigarette smoke and fugitive emissions. Contributions from these sources are expected to be relatively small compared with road vehicles. However, their varying physicochemical characteristics compared with road vehicle induced UFPs can be vital from the exposure and health point of view. Furthermore, their contributions may become apparent in future in the view of stricter particle number emission limits for road vehicles (e.g. Euro 5 and Euro 6 vehicle emission standards) which may lead to possible reduction in release of PNCs from this dominant source as a result of precautionary control measures such as the use of diesel particulate filters or adoption of clean fuel policies. Given the handful of information available in discretely published form, these sources require further research efforts to understand the detailed physical and chemical characteristics of UFPs produced by them and their contributions towards the ambient UFPs.

Concentrations of UFPs can vary by up to five or more orders of magnitude (i.e. from $10^{2} \mathrm{~cm}^{-3}$ in marine environments up to $10^{7} \mathrm{~cm}^{-3}$ closer to vehicle tailpipes), depending on environmental conditions and source strengths [1], so can be the case with associated exposure. Elevated concentrations of airborne UFPs lead to poor air quality along with increasing the probability of human health risks due to exposure to high concentrations. This means that the freshly emitted peak UFP concentrations, occurring in the close vicinity of dominant sources (i.e. road vehicles), are likely to leave the largest negative footprints on the on-road air quality. Let us now focus on the UFP concentration

Corresponding author: Prashant Kumar, Division of Civil, Chemical and Environmental Engineering, Civil Engineering (C5), Faculty of Engineering and Physical Sceinces, University of Surrey, Guildford GU2 7XH, United Kingdom, Tel: +44-0-1483 682762; Fax: +44-0-1483 682135; Email: P.Kumar@surrey.ac.uk or Prashant.Kumar@cantab.net

Received November 18, 2011; Accepted December 10, 2011; Published December 12, 2011

Citation: Kumar P (2011) Footprints of Airborne Ultrafine Particles on Urban Air Quality and Public Health. J Civil Environment Engg 1:e101. doi:10.4172/2165784X.1000e101

Copyright: (C) 2011 Kumar P. This is an open-access article distributed under the terms of the Creative Commons Attribution License, which permits unrestricted use, distribution, and reproduction in any medium, provided the original author and source are credited. 
variations in typical urban settings. Recent reviews on this topic have indicated that exposure to average UFP concentrations can vary between $3 \times 10^{4} \mathrm{~cm}^{-3}$ to $6 \times 10^{4} \mathrm{~cm}^{-3}$ while cycling, walking, travelling in buses, automobiles or ferries [4]. Similar figures were noted after a closer inspection of the data which was computed after the analysis of a total of about 45 sampling locations in 30 different cities within 15 European countries [5]. The average PNCs at the roadside and urban background sites in European environments were found to be $3.82 \pm 3.25 \times 10^{4} \mathrm{~cm}^{-3}$ and $1.63 \pm 0.82 \times 10^{4} \mathrm{~cm}^{-3}$, respectively, indicating an average roadside to background PNC ratio as $\sim 2.4$. As expected due to the presence of road vehicles, the roadside UFP concentrations showed over an order of magnitude ( $\sim 14$ times) difference between the minimum and maximum average PNCs recorded at various urban sites compared with only $\sim 5$ times difference for urban background sites [5]. The differences in maximum and minimum PNCs at an individual urban site can further increase to over 2 orders of magnitude if the short-term peak concentrations are taken into account. This variability in PNCs raises various questions: what should be an appropriate limit value capable of addressing this remarkable spatial variation, generally observed at different locations within an urban area? Should the future control and management strategies target a decrease of PNCs by more than an order of magnitude in urban environments to match the minimum PNCs? Further concerns arises due to the lack of sufficient knowledge on a number of aspects such as exposure-response relationships, standardisation of the key measurement parameters (including sampling necessary for robust evaluation of PNCs), repeatability and reproducibility in measurements due to the absence of standardisation of instruments, physicochemical characteristics of emerging sources (i.e. bio-fuel derived and manufactured nanoparticles, though the latter is more important for indoor laboratory workplaces), fit for purpose dispersion modelling tools capable of treating varying nature of transformation processes at various spatial scales, and longterm monitoring studies for evaluating the performance of dispersion models which can have the ability to evaluate available knowledge, assess alternative options and regulatory compliance.

Recent estimates report an average loss of 7-8 months in life expectancy to UK residents due to $\mathrm{PM}_{25}$ exposure, with equivalent health costs of $\sim £ 20$ billion per year. Such figures are not available separately for UFP pollution, but an equivalent or greater health costs could be expected given the exposure-response coefficients which are rarely available for the UFPs. These coefficients relate a change in PNCs to the number of associated deaths and are expected to vary for different geographic locations due to the change in social conditions (e.g. food, sanitation, medical care). One of the first studies on this topic made preliminary estimates of ambient UFP exposure related excess deaths in megacity Delhi [6]. A notable number of excess deaths were reported: $\sim 508$ and $\sim 1888$ deaths per million people in 2010 and 2030, respectively, under the business as usual scenario. Further, normalization of the above mortality figures provided $\sim 0.69$ and 48 times relative mortality impact by vehicle-derived UFPs in Delhi compared with the TSP and $\mathrm{NO}_{2}$ exposure occurring from all sources, respectively. More identical studies are needed for megacities in worldwide but the location specific particle number emission factors and exposure-response coefficients are major constraints for such computations and warrant further research.

Outdoor UFPs can also enter into the indoor environments such as residential and commercial buildings, offices or restaurants via the air infiltration or ventilation system. In addition, presence of already existing indoor sources during operation can increase the exposure to short-term UFP concentrations up to an order of magnitude or larger than those found in outdoor environments. Some of the key indoor sources include cooking using gas, electric stoves or toasters, hair dryers, wax candles, fire places, electric irons and mixers, cleaning, air freshener sprays or tobacco smoke, leaving aside those originating from such as the laser printers in offices and combustion of solid fuels (e.g. wood, dung, crop residue or coal) inside houses in developing countries. Although most of the indoor sources are non-continuous but the exposures can still be significant considering that up to $90 \%$ of the total daily time is typically spend indoors compared with only about $6 \%$ outdoors. Indoor concentrations normally reach to their maximum in a few minutes just after the activation of the source. After the switching off indoor sources, concentration decay generally occurs at a much slower rate due to the surrounding built up environment restricting the ventilation and dilution. A recent study, based on suburban areas in USA, characterised the indoor sources in detail [7]. They estimated relative contribution to the typical $24 \mathrm{~h}$ daily nonsmoker style exposures by outdoor, indoor and in-vehicle sources as 36,47 and $17 \%$, respectively. This contribution was doubled for indoor (77\%), nearly halved for outdoor (17\%) and one-third for in-vehicle $(6 \%)$ in the presence of a smoker smoking on an average 16 cigarettes per day in an averaged size house having volume of $400 \mathrm{~m}^{3}$ and typical air exchange rates of about $0.75 \mathrm{~h}^{-1}$ [7]. As expected, the exposure contribution from outdoor sources can decrease in rural areas and increase in urban areas due to a greater mobility of road vehicles. For instance, the above figures for non-smoking case were found to be swapped by each other in a Los Angles based urban study, with about $46 \%, 36 \%$ and the reminder exposure occurring in outdoor, in-vehicle and indoor environments, respectively [8].

One of the highest priorities of the European Union's energy policy includes improvement in energy efficiency of buildings. Reduction in air leakage rates is among one of the measures for designing such strategies. Such measures are effective in reducing the penetration of outdoor air pollutants into the buildings and for energy savings. However, the reduced air exchange rates may lead to a build up of pollutant concentrations, including of UFPs, and worsen the air quality of indoor environments if such policies are not properly assessed before implementation. As for outdoor environments, there are no indoor air quality guidelines for controlling UFP levels. Much of the existing control guidelines target the laboratory or workplaces environments for engineered nanoparticles [9], but not the exposure to UFPs generated in residential indoor settings. Similar to the outdoor UFPs, mitigation strategies are constraint by indecisive answers of several questions. For instance, it is yet inconclusive that what levels of UFP exposure should be safe and acceptable for different indoor environments. Whether these levels should be the same for all buildings and how should exposure to peak concentrations be treated? Further concerns can be raised on the currently available means to distinguish the contribution of different sources from the background. Moreover, there is limited information available in patches on the penetration of outdoor UFPs into the indoor environments. This makes difficult to derive generalised indoor to outdoor ratios for UFPs and computing the influence of air infiltration on these ratios for different building types is even harder. Also is not very well studied the role of transformation processes (e.g. nucleation, dry deposition, coagulation) in indoor environments and how does these alter the size resolved distributions and concentrations of UFPs under both conditions when indoor sources are switched on or off. Due to a wide range of indoor sources, physical and chemical characteristics of UFPs generated can be different than those generated outdoors. This may further complicate the already weak exposureresponse links between the UFPs and the public health. Dedicated studies are also needed to assess the influence of energy consumption in new energy-efficient, modified or existing buildings on the UFP exposure for designing sustainable solutions. 
Citation: Kumar P (2011) Footprints of Airborne Ultrafine Particles on Urban Air Quality and Public Health. J Civil Environment Engg 1:e101. doi:10.4172/2165-784X.1000e101

Despite the evidences showing negative footprints of UFPs on urban air quality and public health, envisaging mitigation strategies for both indoor and outdoor UFPs still appears to be a way-off, at least until the answers of some of the aforementioned key questions is known through the further field, laboratory or modelling studies. Given the rapidly evolving nature of this inter-disciplinary research topic, fast dissemination of research, accessible freely to a wider audience, is crucial. The open access journals could be one such platform for rapid transmission of research on the current and the other equally important topics.

\section{Brief bio of the Contributing Author}

Dr. Prashant Kumar is a Lecturer in Civil and Environmental Engineering at the University of Surrey, UK. Main focus of his research is on measurements of airborne nano/ultrafine particles originating from various combustion and nontraffic sources in different urban settings using the state-of-the-art advanced instruments and dispersion modelling by combining the key findings from field, wind tunnel and computational studies.

\section{References}

1. Kumar P, Robins A, Vardoulakis S, Britter R (2010) A review of the characteristics of nanoparticles in the urban atmosphere and the prospects for developing regulatory controls. Atmos Environ 44: 5035-5052.
2. Kumar P, Ketzel M, Vardoulakis S, Pirjola L, Britter R (2011) Dynamics and dispersion modelling of nanoparticles from road traffic in the urban atmospheric environment - a review. J Aerosol Sci 42: 580-603.

3. Kumar P, Robins A, Vardoulakis S, Quincey P (2011) Technical challenges in tackling regulatory concerns for urban atmospheric nanoparticles. Particuology 9: $566-571$

4. Knibbs LD, Cole-Hunter T, Morawska L (2011) A review of commuter exposure to ultrafine particles and its health effects. Atmo Environ 45: 2611-2622.

5. Kumar P, Morawska L, Harrison RM (2012) Nanoparticles in European cities and associated health impacts. The Handbook of Environmental Chemistry: Air Quality in Europe (Springer Eds.), Volume editor Dr. Mar Viana. (invited under review)

6. Kumar P, Gurjar BR, Nagpure A, Harrison RM (2011) Preliminary estimates of nanoparticle number emissions from road vehicles in megacity Delhi and associated health impacts. Environ Sci Technol 45: 5514-5521.

7. Wallace L, Ott W (2011) Personal exposure to ultrafine particles. J Exposure Sci \& Environ Epid 21: 20-30.

8. Fruin S, Westerdahl D, Sax T, Sioutas C, Fine PM (2008) Measurements and predictors of on-road ultrafine particle concentrations and associated pollutants in Los Angeles. Atmos Enviro 42: 207-219.

9. Kumar P, Fennell P, Robins A (2010) Comparison of the behaviour of manufactured and other airborne nanoparticles and the consequences for prioritising research and regulation activities. J Nano Res 12: 1523-1530. 